\title{
Early SUSY searches at the LHC
}

\author{
A. D. Tapper* on behalf of the ATLAS and CMS Collaborations \\ Imperial College London \\ E-mail: a.tapper@imperial.ac.uk
}

Supersymmetry may give rise to striking events that could be discovered early in LHC running. Search strategies based on the generic event signatures of high jet multiplicity and large missing transverse momentum, optionally including leptons in the final state, are discussed. A highly important aspect of such searches is the prediction of Standard Model backgrounds using datadriven methods, which is discussed in detail. Finally prospects for a discovery in early LHC running are reviewed.

XXth Hadron Collider Physics Symposium

November 16 - 20, 2009

Evian, France

* Speaker. 


\section{Introduction}

The Large Hadron Collider (LHC) [1] at CERN offers enormous potential to discover new physics beyond the Standard Model. In this paper strategies for searching for one of the most favoured theories beyond the Standard Model, Supersymmetry (SUSY) are discussed. Many different SUSY scenarios have been investigated by the ATLAS [2] and CMS experiments in the past (see [4] and [5] for example). This paper describes what could be achieved in early running at a cente-of-mass energy $(\sqrt{s})$ of $10 \mathrm{TeV}$ with $100-200 \mathrm{pb}^{-1}$ of data. Particular emphasis is given to the development of data-driven estimates of the Standard Model background contributions.

\section{Search strategy}

While searches should be as model independent as possible the large number of free parameters in many SUSY models makes this a challenging task. The approach taken by the ATLAS and CMS collaborations has been to choose a set of benchmark points, from a constrained model, which are representative of a range of event topologies and areas of phase space. Benchmark points [4] [5] have been defined in several models including mSUGRA, GMSB and split SUSY. This paper uses mSUGRA benchmark points just above the discovery reach of the Tevatron experiments, to illustrate the discovery reach in early running.

At LHC energies squark and gluino production are expected to dominate SUSY particle production. Since squarks and gluinos are coloured particles they are predicted to be produced via the stong interaction with a cross section similar to that of QCD processes at the same scale. To a good approximation the production cross sections depend only on the masses of the particles and are therefore independent of the SUSY model. In contrast the decays of the SUSY particles depend critically on the details of the SUSY model under consideration, such as mass spectra and branching ratios. However some generic properties can be identified. Assuming R-parity is conserved then the decay chains end with the lightest SUSY particle which escapes undetected, leading to missing transverse momentum (MET). Assuming that the squarks and gluinos are heavy, long decay chains are expected, with several jets or leptons involved.

In summary the search strategy of both the ATLAS and CMS collaborations is to focus on simple and robust event signatures, which are common to a wide variety of models and let the Standard Model backgrounds and the performance of the detectors define the search ranges.

\section{Searches}

A generic search of the type described above can be constructed using simple event selections to categorise events with large MET by numbers of leptons and jets [6]. An excess of events from many SUSY benchmark signals can easily be observed in many event topologies with as little as $200 \mathrm{pb}^{-1}$ of data, taken at $\sqrt{s}=10 \mathrm{TeV}$. It is not advisable to assume that the Standard Model backgrounds are well predicted by Monte Carlo, so the key to such searches will be to measure the background contributions directly from the data.

Backgrounds to SUSY signal events can be catagorised roughly into three types. Firstly, detector effects such as noise, mis-measurements or poorly calibrated regions of a detector usually 
lead to fake MET. A high level of control over data quality and calibration is required to understand and reduce such effects.

Secondly, beam-related backgrounds such as muons produced by the beam halo or from cosmic rays, and interactions between the beams and residual gas in the beam pipes can also lead to fake MET in otherwise well balanced collision events. These effects can be tackled with a combination of simulation and and in-situ measurements.

Lastly, physics backgrounds are Standard Model processes that give the same signatures as SUSY. Generally Monte Carlo predictions should not be relied upon since these come from tuning to lower energy data and typically SUSY events also lie in problematic areas of phase space, such as large numbers of jets, where Monte Carlo simulations are known not to give high-precision predictions. These processes should instead be measured from the data itself.

The general idea in order to measure the physics backgrounds from the data is to find a control region where the Standard Model is dominant and use this to predict the background in a signal region. An often pursued approach is to find a pair of variables that are both sensitive to the signal and play them off against eachother to predict the background in the signal region. Another much used approach is to modify Standard Model events with the same topology as the signal to predict the background in the signal region. In both cases the need to identify a clean control region is difficult and, as will be discussed it is difficult to avoid using Monte Carlo simulation in some way.

The following sections will discuss different search channels giving examples of data-driven methods and discussing sensitivity to new physics.

\subsection{Hadronic searches}

Searches based on only jets and MET are highly sensitive to SUSY but suffer from many backgrounds, making them excellent examples to illustrate data-driven background methods.

Mis-measurement of one or more jets in otherwise balanced QCD events leads to MET and forms a background to SUSY searches. Several methods have been developed to predict the MET tail from QCD events, here one such method is described. The technique [7] uses a control sample of events with a prompt photon and jets to derive a core Gaussian component of a jet mismeasurement function, a sample with three well separated jets to derive a non-Gaussian tail and a di-jet sample is used to normalise the Gaussian and non-Gaussian parts. This smearing function is then applied to low-MET events to predict the tail in the high-MET signal region. Figure 1 shows the derived smearing function and a Monte Carlo closure test where the predicted background contamination is compared to the true contamination, suggesting the method should work.

A novel approach [8] to control QCD background using both angular and energy information has been proposed, originally for di-jet events [9] and subsequently for higher numbers of jets [10]. The technique defines a variable $\alpha_{\mathrm{T}}=\frac{E_{\mathrm{T}}^{\mathrm{j} 2}}{M_{\mathrm{T}}^{\mathrm{j}, \mathrm{j} 2}}$, where $E_{\mathrm{T}}^{\mathrm{j} 2}$ is the transverse energy of the sub-leading jet in a di-jet event and $M_{\mathrm{T}}^{\mathrm{j} 1, \mathrm{j} 2}$ is the transverse mass of the di-jet system. One can easily see that for a well-measured di-jet event $\alpha_{\mathrm{T}}$ should be 0.5 and mis-measurements lead to lower values. Figure 2 show the distribution of $\alpha_{\mathrm{T}}$ for background and SUSY signal events. It can been seen that a strong separation between QCD background and SUSY signal can be achieved, critically without use of the MET variable, making this technique highly robust and ideally suited to early data. 

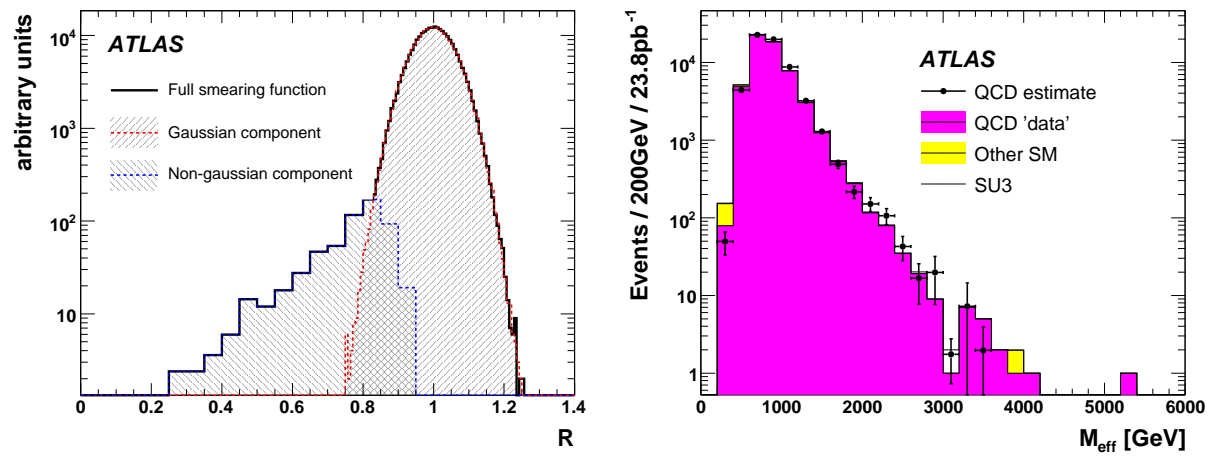

Figure 1: The jet smearing function (left) and closure test of the QCD background estimation (right).
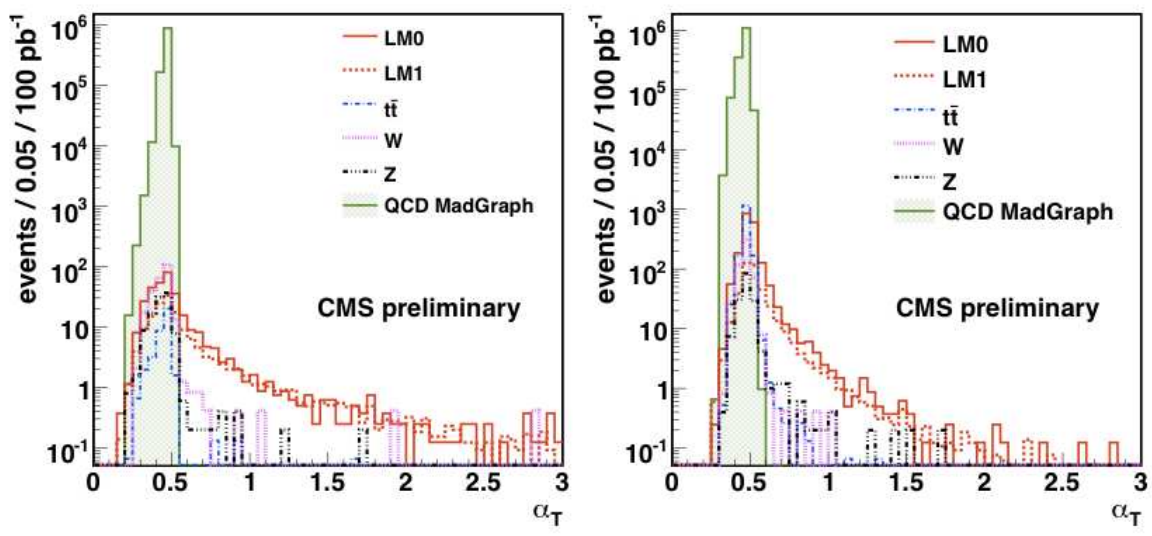

Figure 2: The $\alpha_{\mathrm{T}}$ distribution for di-jet events (left) and three to six jet events (right).

\subsection{Single-lepton searches}

Requiring the presence of an electron or muon in the search criteria suppresses QCD background powerfully and maintains high sensitivity to SUSY signals. Backgrounds then come from Standard Model processes with neutrinos leading to real MET, in particular top-quark and $W$-boson decays. Many methods have been developed to evaluate background contamination in such events, here one of the latest is discussed.

The "Tiles" method [11] builds on the well known " $M_{\mathrm{T}}$ " method [7] in which a low-MET low- $M_{\mathrm{T}}$ control region is used to predict the top-quark and $W$-boson backgrounds in the high-MET signal region. Some weaknesses of the $M_{\mathrm{T}}$ method are that it breaks down when the MET and $M_{\mathrm{T}}$ variables are not independent and potentially suffers from signal contamination in the background control region. The newer Tiles method mitigates these weaknesses at the expense of increased use of Monte Carlo simulation. Using a similar pair of variables, $M_{\mathrm{T}}$ and $M_{\mathrm{eff}}$ (the scalar sum of the jet and lepton $E_{\mathrm{T}} \mathrm{s}$ and MET in the event), control and signal regions are defined. The number of events in each region can be expressed with a system of linear equations, which may be solved using the Monte Carlo prediction for the shapes of the Standard Model backgrounds and assuming 
the independence of the variables for signal events. The solution predicts the number of background and SUSY signal events in each region and importantly the background prediction not biassed by signal contamination in the control region.

\subsection{Di-lepton searches}

Searches for SUSY in the di-lepton channels have low yields but very interesting properties. Searches where both leptons have the same sign are particularly interesting since they have a very low Standard Model background rate, stemming only from charge mis-identified top-quark events and inefficiency in isolation criteria when vetoing semi-leptonic heavy-flavour decays within jets. In addition the opposite-sign, opposite-flavour samples provide a reliable control region to subtract the Standard Model background.

Addtionally di-lepton events can provide information on the masses of SUSY particles through the end-point mass of the di-lepton system. Figure 3 [12] shows an example of such an analysis where the $e e, \mu \mu$ and $e \mu$ distributions are fitted to obtain the efficiencies, resolutions and end-point mass from the data with $200 \mathrm{pb}^{-1}$ of data at $\sqrt{s}=10 \mathrm{TeV}$. In this example the di-leptonic end-point is measured to be $m_{l l}=51.3 \pm 1.5$ (stat.) \pm 0.9 (syst.) $\mathrm{GeV}$ in good agreement with the generated value of $52.7 \mathrm{GeV}$. This is a nice example of what may be achieved with a modest dataset.
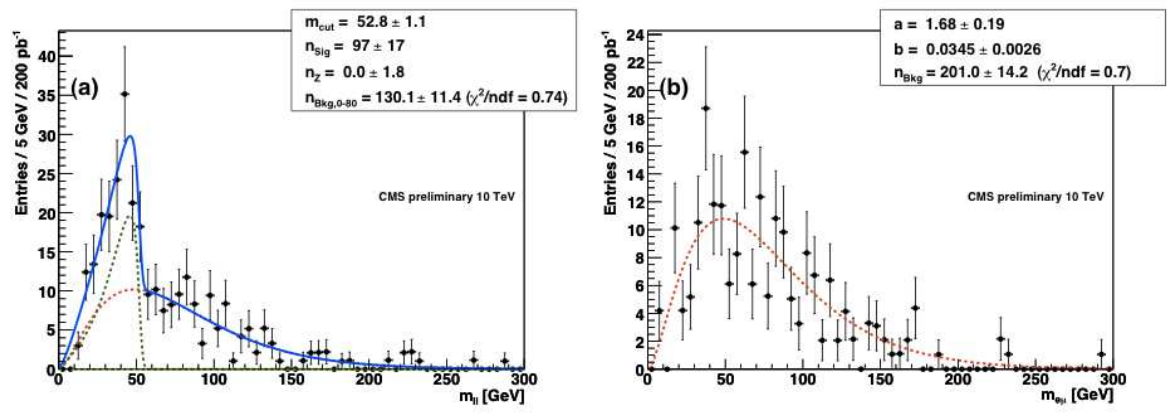

Figure 3: The combined fit to the di-lepton end-point is shown in (a). The green curve represents the SUSY signal model, the red curve is the background function and the light green dashed line the Z-boson contribution. The black points represent the MC events. In (b) the fit of the background function to the $e \mu$ invariant mass distribution is shown.

\section{Discovery reach}

The prospects for the discovery reach are studied with $200 \mathrm{pb}^{-1}$ of simulated data at $\sqrt{s}=$ $10 \mathrm{TeV}$ and are shown in the mSUGRA model in Fig. 4 [6]. It can be seen that the 4 jet 0 lepton channel and the 4 jet 1 lepton channel are the most sensitive searches. Both of these channels give clear discovery reach beyond that of the Tevatron experiments.

\section{Summary}

The CMS and ATLAS collaborations have developed searches based on robust generic event signatures, suitable to early LHC running and sensitive to a variety of new physics models. A 

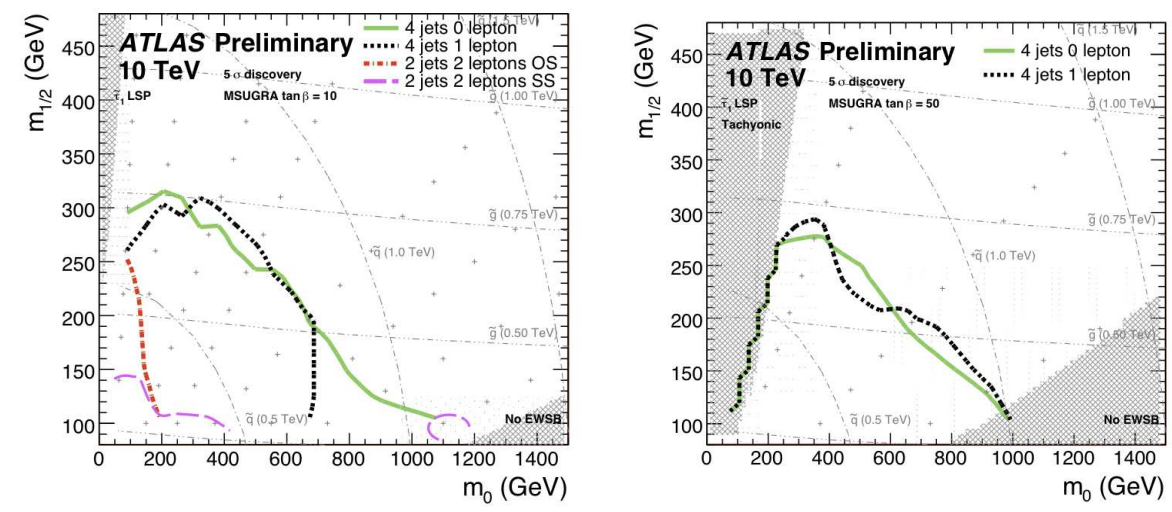

Figure 4: The $5 \sigma$ discovery contours for the 4 jet 0 lepton, the 4 jet 1 lepton and the 2 jet 2 lepton channels as a function of $\mathrm{M}_{0}$ and $\mathrm{M}_{1 / 2}$ for the mSUGRA model with $\tan \beta=10$ (left) and $\tan \beta=50$ (right).

wide range of data-driven techniques have been developed to measure efficiencies and backgrounds from the collision data.

\section{References}

[1] L. Evans \& P. Bryant, LHC Machine, JINST3:S08001 (2008).

[2] ATLAS Collaboration, The ATLAS Experiment at the CERN LHC, JINST3:S08003 (2008).

[3] CMS Collaboration, The CMS experiment at the CERN LHC, JINST3:S08004 (2008).

[4] ATLAS Collaboration, Detector and Physics Performance Technical Design Report, LHCC 99-14/15 (1999).

[5] CMS Collaboration, CMS Physics Technical Design Report Volume II: Physics Performance, J. Phys. G: Nucl. Part. Phys. 34 (2006).

[6] ATLAS Collaboration, Prospects for Supersymmetry and Univeral Extra Dimensions discovery based on inclusive searches at a $10 \mathrm{TeV}$ centre-of-mass energy with the ATLAS detector, ATL-PHYS-PUB-2009-084 (2009).

[7] ATLAS Collaboration, Expected Performance of the ATLAS Experiment, Detector, Trigger and Physics, CERN-OPEN-2008-020 (2008).

[8] L. Randall \& D. Tucker-Smith, Phys. Rev. Lett. 101:221803 (2008).

[9] CMS Collaboration, SUSY searches with dijet events, CMS-PAS-SUS-08-005 (2008).

[10] CMS Collaboration, Search strategy for exclusive multi-jet events from supersymmetry at CMS, CMS-PAS-SUS-09-001 (2009).

[11] ATLAS Collaboration, Background Estimation for Inclusive SUSY Searches - The Tiles Method, ATL-PHYS-PUB-2009-077 (2009).

[12] CMS Collaboration, Discovery potential and measurement of a dilepton mass edge in SUSY events at $\sqrt{s}=10 \mathrm{TeV}$, CMS-PAS-SUS-09-002 (2009). 